\title{
A Prospective Analysis of Otomycosis in a Tertiary Care Hospital
}

\section{Shuaib Kayode Aremu ${ }^{1 *}$, Kayode Rasaq Adewoye ${ }^{2}$ and Tayo Ibrahim ${ }^{3}$}

\author{
${ }^{1}$ Department of Ear, Nose and Throat, Federal Teaching Hospital, Ido-Ekiti/Afe Babalola University, Nigeria \\ ${ }^{2}$ Department of Community Medicine, Federal Teaching Hospital, Ido-Ekiti/Afe Babalola University, Nigeria \\ ${ }^{3}$ Department of Ophthalmology, Federal Teaching Hospital, Ido-Ekiti/Afe Babalola University, Ado-Ekiti, Ekiti \\ State, Nigeria
}

*Corresponding author: Shuaib Kayode Aremu, ENT Department, Federal Teaching Hospital, Ido-Ekiti/Afe Babalola University, Ado-Ekiti, Ekiti State, Postal Code: 371101, Nigeria, Tel: +2348033583842 , Fax: +2348033583842

\begin{abstract}
Background: Otomycosis is a fungal infection of the external auditory canal, commonly encountered in the general otolaryngology department. Otomycosis is more frequently observed in hot and humid climates and various individual, as well as environmental factors, predispose to this infection. This study aims to explore the prevalence of otomycosis in a tertiary care hospital in Ekiti state, Nigeria, along with a focus on patient demographics, predisposing risk factors, and pathogenic causes.
\end{abstract}

Materials and methods: The study was conducted in the Ear, Nose, and Throat (ENT) department of Federal Teaching Hospital Ido-Ekiti, which is a tertiary care hospital in Ekiti state, Nigeria. The study involved 275 patients above the age of 10 , who presented in the ENT department over 5 years period and were clinically diagnosed cases of otomycosis. Certain patient demographics such as age, gender, and clinical presentations were recorded along with possible risk factors and clinically observed findings. Microbiological causes were evaluated via the culture of samples collected from each individual. Results were statistically analysed and the results are presented in a tabulated manner.

Results: The results obtained in our study revealed that the majority of the subjects were between the ages of 20 and 30 years, making a total of $38.2 \%$ of the study population. Males were observed more commonly then females accounting for $56 \%$ of the total study population. 267 patients reported unilateral ear involvement among which the right ear was involved in $82 \%$ of the cases. The most frequently observed risk factor was self-cleaning of ears with objects such as q-tips, wooden sticks, metal pickers, etc. seen in $71 \%$ of the study population, followed by the use of antibiotic ear drops observed in $57 \%$ and instillation of mustard oil in $52 \%$ of the study group. The most common presenting symptom in the study population was reported to be pruritus of the ear in $73 \%$ followed by otalgia in $66.5 \%$, and sensation of blocked ear observed in $57 \%$. Fungi were established to be the causative organism in 270 out of the total 275 samples and the most commonly isolated fungi were Aspergillus seen in $91 \%$ of the total population. The most common species of Aspergillus that was isolated from samples was Aspergillus Niger seen in $56 \%$. The second most commonly isolated fungus was Candida in $13.8 \%$ of the group. Bacteria were isolated from $56.4 \%$ of the total samples as a concomitant organism, Staphylococcus aureus is the most commonly seen in $58 \%$ of the samples. The present study, apart from highlighting the patient population and causative agents of otomycosis, also places stringent focus on the need for education to eradicate the aforementioned predisposing risk factors to hinder the incidence of otomycosis.

\section{Keywords}

Otomycosis, Otitis externa, Fungal ear infections, Candida, Aspergillus, Microbiological causes, Risk factors

\section{Introduction}

Concerning ear complain related visits to Ear, Nose, and Throat (ENT) departments, it is estimated that otitis externa makes up about 5 to $20 \%$ of the total clinic presentations, and among the total, approximately 10 to $25 \%$ can be attributed to fungal infections broadly referred to as fungal otitis externa or otomycosis $[1,2]$. Otomycosis is commonly observed in tropical and subtropical regions, although it is prevalent globally $[3,4]$. Otomycosis involves the squamous epithelium of the external ear canal and most frequently the causative fungi occupy the medial aspect of the ear canal, partly owing to the location of the inferior tympanic recess that permits the accumulation of debris and partly due to this aspect of the ear canal being comparatively,

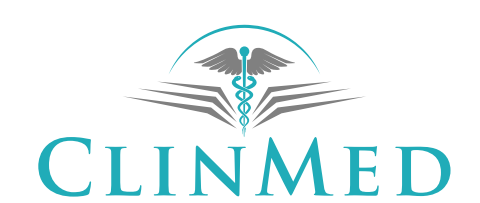

INTERNATIONAL LIBRARY

Citation: Aremu SK, Adewoye KR, Ibrahim T (2020) A Prospective Analysis of Otomycosis in a Tertiary Care Hospital. Int J Trop Dis 3:029. doi.org/10.23937/2643-461X/1710029

Accepted: April 06, 2020; Published: April 08, 2020

Copyright: (C) 2020 Aremu SK, et al. This is an open-access article distributed under the terms of the Creative Commons Attribution License, which permits unrestricted use, distribution, and reproduction in any medium, provided the original author and source are credited. 
darker and warmer, thus promoting fungal growth [5]

Patients with otomycosis commonly present with complaints of ear itching, discharge, discomfort, and/ or a feeling that something is in the ear canal. Patients may also complain of otalgia, ear fullness and ear blocking sensation [4]. In some cases, hypoacusis, tinnitus and/or hearing impairment may also be reported $[6,7]$. Deep-seated itching in the ear canal is often reported to be the most troublesome symptom; pain, however, is reported to be less intense when compared to bacterial external otitis.

Several hosts and environment-related factors increase the likelihood of acquiring otomycosis. These include local ear-related factors such as changes in $\mathrm{pH}$, changes in the ear's epithelium, and quantitative as well as qualitative changes in ear wax [8]. Additionally, bacterial infection, instrumentation of the ear, use of hearing aids, self-inflicted trauma such as with the use of q-tips, and swimming all predispose to otomycosis [9]. Systemic factors that enhance the risk of otomycosis include the long term use of steroids, cytostatic medications or broad-spectrum antibiotic agents, cancer and other immune disorders, all of which depreciate the immune system and render the host susceptible to otomycosis [3].

Fungi rarely cause disease in healthy immunocompetent hosts. The disease occurs when fungi by accident penetrate host barriers or when immunologic defects or other debilitating conditions exist that favor fungal entry and growth. Fungi often develop both virulence mechanisms (e.g., capsule and ability to grow at $37{ }^{\circ} \mathrm{C}$ ) and morphologic forms (e.g., yeasts, hyphae, spherules, and sclerotic bodies) which enhance their multiplication within the host. Dissemination of fungi in the body indicates a breach or deficiency of host defenses (e.g., endocrinopathies and immune disorders). Host factors are also responsible for easy affectation by fungi. Healthy, immunologically-competent individuals have a high degree of innate resistance to fungi. Resistance to fungi is based primarily upon cutaneous and mucosal physical barriers. In addition, fungal factors are implicated and certain enzymes have been found to play a major role. keratinase Enzyme, the capsule in Cryptococcus neoformans, the ability to grow at $37^{\circ} \mathrm{C}$, dimorphism, and some other yet to be identified factors contribute to fungal pathogenesis which involves a complex interplay of many fungal and host factors.

The most commonly implicated organisms in otomycosis are species from the fungi genera of Aspergillus and Candida [10]. Aspergillus is a ubiquitous mold identified as the causative organism in various infections and diseases. Aspergillus can be found in the form of minute conidia that can be easily propelled into the air with dust and other particles, making this its primary route of transmission.

Examination of the ear canal is usually done through otoscopy and biomicroscopy, while confirmation is obtained through mycological exams. Aspergillus and Candida are opportunistic organisms found as a component of the normal microbiota of various body parts [11]. Upon visualization, Aspergillus Niger spores appear like fine coal dust sprinkled in the ear canal. They may also resemble a blotting paper or a crumpled newspaper. On the other hand, Candida infections of the external ear are seen as white, cheesy, sebaceous-like material that may in severe cases, even fill the ear canal. In such cases, a pseudomembrane often lines the ear canal, the removal of which reveals an underlying granular and friable membrane [12].

Treatment options for otomycosis range from control of underlying predisposing conditions to causative pathogen termination, to local debridement i.e. microaspiration and/or the use of topical or systemic antimicrobial agents [13].

Several studies and analyses have been conducted on otomycosis, around the globe but few reported studies exist in the region of Kwara state of Nigeria. The present paper aims at assessing otomycosis concerning the affected patient population, and its demographics as well as the presenting symptoms and pathogenic causes of otomycosis in this region.

\section{Material and Methods}

\section{Patient population}

The study was performed in the Ear. Nose and throat (ENT) department of Federal Teaching Hospital Ido-Ekiti state, Nigeria. The study included 275 patients who presented to this department over 5 Years from July 2013 to July 2018. The patients included in this prospective analysis were carefully chosen given the criteria below.

\section{Inclusion criteria}

Only patients that were above the age of 10 were included in the study. Only patients who were diagnosed with otomycosis based upon history and physical examination were included in this analysis. The symptoms used to diagnoses otomycosis included pruritus of the ear, otalgia, hearing impairment, tinnitus, discharge and sensation of having an ear blockage. On examination, otoscopic findings were used to support the clinical diagnosis of otomycosis to aid the selection of patients for study such as soft, white, cheese-like sebaceous material or finely sprinkled or matted masses of hyphae and/or spores [14].

\section{Exclusion criteria}

All patients below the age of 10 were excluded from the study. Patients with a history of serious, chronic medical illnesses such as tuberculosis and diabetes were excluded from the study. Screening for these conditions was done via relevant medical histories, records as well as Purified Protein Derivative (PPD) test for tuberculosis 
and fasting blood glucose levels for diabetes. Patients who had a history of ear surgery, tympanic membrane perforations due to barotrauma, and recurrent otitis media both acute and chronic variety were excluded from the study.

\section{Data and sample collection}

Data were collected from each of the 275 patients via complete and detailed history taking, physical examination including otoscopy of the ear, investigation with swab sample collections and the obtained results then complied. During history taking, various patient demographics were recorded such as age, gender, occupation, socioeconomic factors along with the presence of any risk factors that may predispose to otomycosis such as the use of long term antibiotic ear drops, use of q-tips or wooden sticks or metal pickers for ear cleaning or the use of mustard oil instilled in the ear canal and swimming. Clinical presentations of each of the patients were also recorded including unilateral or bilateral ear involvement. Following history taking, otoscopy was done to evaluate for the presence of white cheesy material or finely granular wet or dry matted spores of both the affected and normal ear for comparison purposes. Following examination, samples were collected from the affected ear canal only, using sterile swabs with a curette and a cotton carrier. Each of the samples obtained was sent to the laboratory within the hospital as well as a private laboratory within the state as soon as possible usually within the first hour of obtaining the samples. Following the collection of samples for mycobacterial analysis, each of the 275 patients received a complete ear toilet, along with a prescription of clotrimazole with instructions to instill a few drops once every day for 2 weeks. Follow up visit was set up after 2 weeks.

Samples were assessed in the laboratory by direct examination as well as culture on blood and MacConkey agar for $48 \mathrm{hr}$. Growth of bacterial colonies was noted and fungal growth was identified by using $10 \%$ potassium hydroxide $(\mathrm{KOH})$ solution. If fungal growth was observed, it was inoculated on standard Sabouraud's Dextrose Agar (SDA) with chloramphenicol which was incubated under aerobic conditions at both room temperature of $25^{\circ} \mathrm{C}$ and body temperature of $37^{\circ} \mathrm{C}$ for the duration of one month. The presence of fungal colonies on the agar was checked every 3 days while colonies were identified using observation of morphology as well as Lactophenol Cotton Blue (LPCB) mount microscopy. Aspergillus was identified through the different length of conidiophores while Candida was identified using the standard, germ tube test, while the specific species was characterized by HiChrome agar.

\section{Statistical analysis of data}

Data were presented in the form of tables and charts using percentages of the total population. Chi-square test was used to find the association between risk fac- tors and otomycosis with $p$-value 0.05 being set as the cut off for significance.

\section{Results}

The total study population comprised of 275 subjects, among which the maximum number of subjects were between the ages of 20 and 30 years, making a total of $38.2 \%$ of the study population. The Mean \pm SD $=27.8 \pm 11.0$. The second most commonly encountered age group was between 10 and 20 years that comprised of 78 patients. The least number of patients belonged to 50 and above group making only $3.6 \%$ of the total population (Table 1). $56 \%$ of the study population was males and $44 \%$ were females, equivalent to a number of 154 males subjects and 121 females subjects (Figure 1).

In 267 patients, only unilateral ear involvement was observed, which was reported to be the right in $82 \%$ and the left ear in $18 \%$ of the cases. $3 \%$ of the study population reported bilateral ear involvement.

All patients were asked questions to establish the presence of any predisposing risk factors. The most prevalent risk factor observed in the study population was self-cleaning of ears with objects such as q-tips, wooden sticks, metal pickers, hairpins, etc. This was observed in $71 \%$ of the study population. The second most

Table 1: Age distribution of study subjects.

\begin{tabular}{|l|l|l|}
\hline Age (years) & Number $(\mathbf{N}=\mathbf{2 7 5})$ & $\begin{array}{l}\text { \% of Total } \\
\text { Population }\end{array}$ \\
\hline $10-20$ & 78 & 28.4 \\
\hline $20-30$ & 105 & 38.2 \\
\hline $30-40$ & 54 & 19.6 \\
\hline $40-50$ & 28 & 10.2 \\
\hline 50 and above & 10 & 3.6 \\
\hline
\end{tabular}

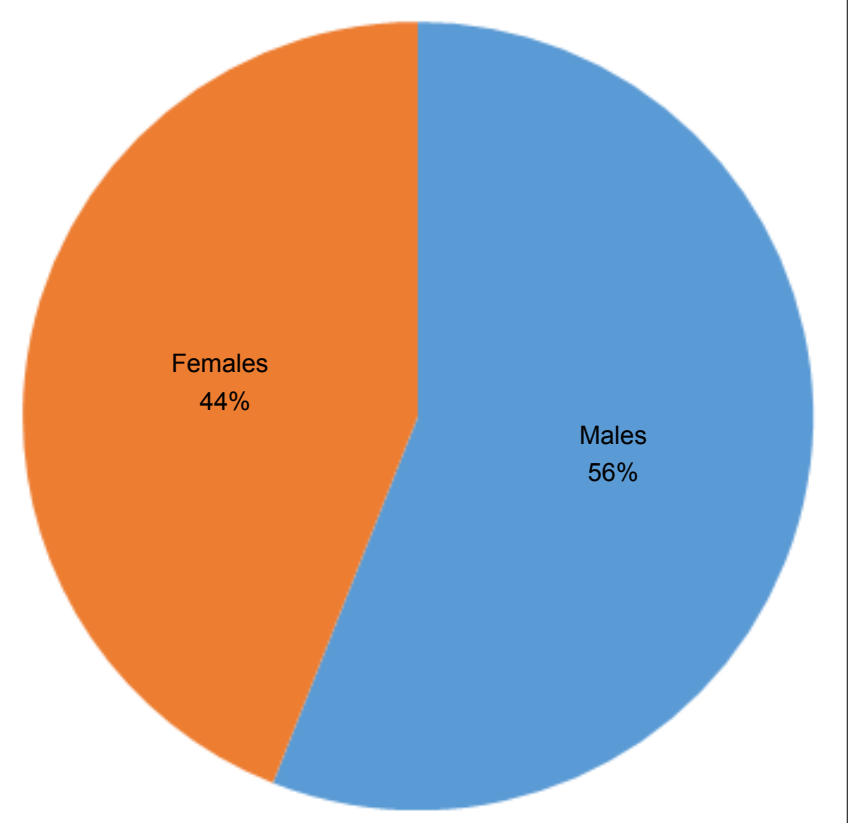

Figure 1: Gender distribution of study subjects. 


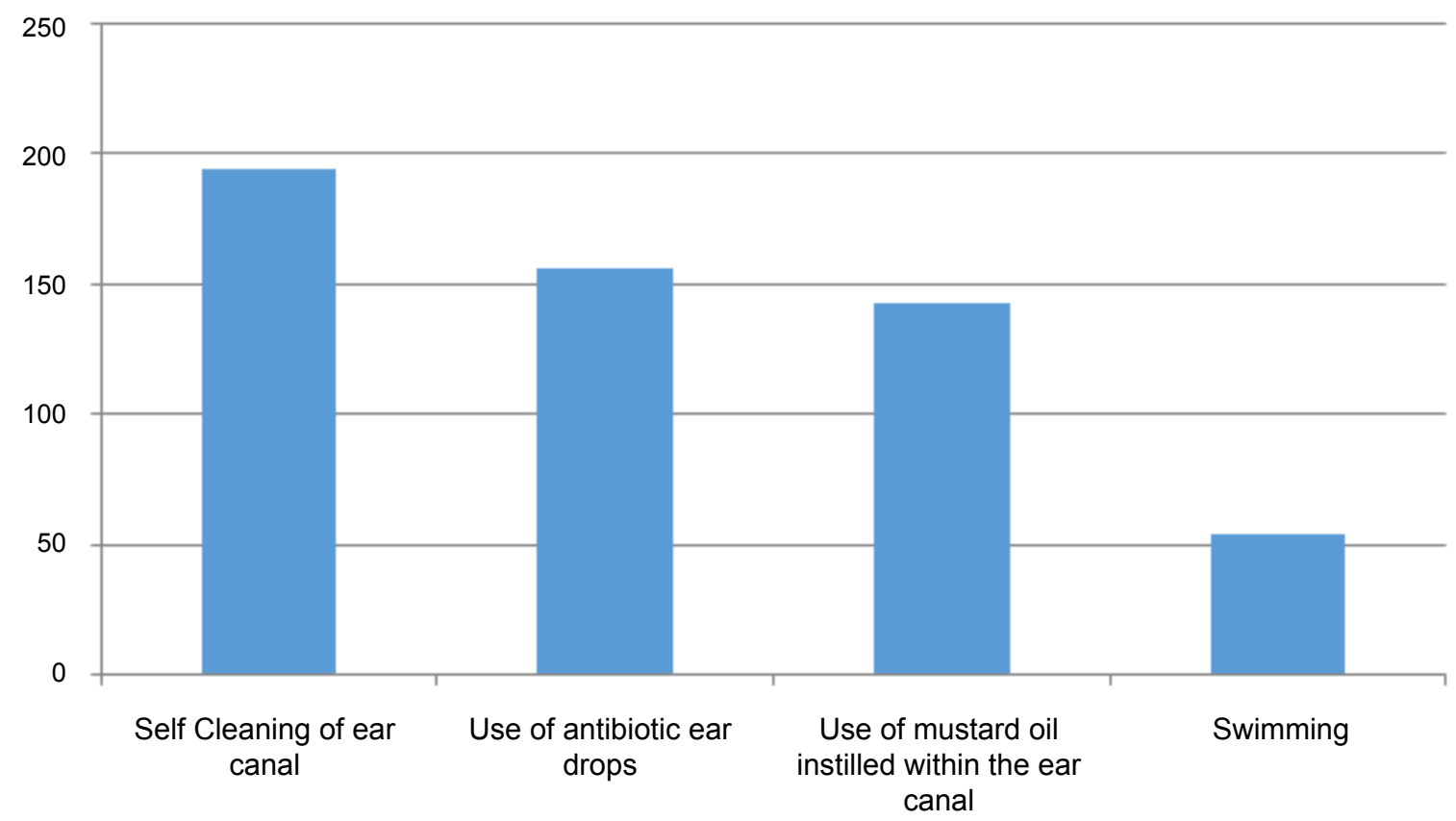

Figure 2: Risk factors associated with otomycosis.

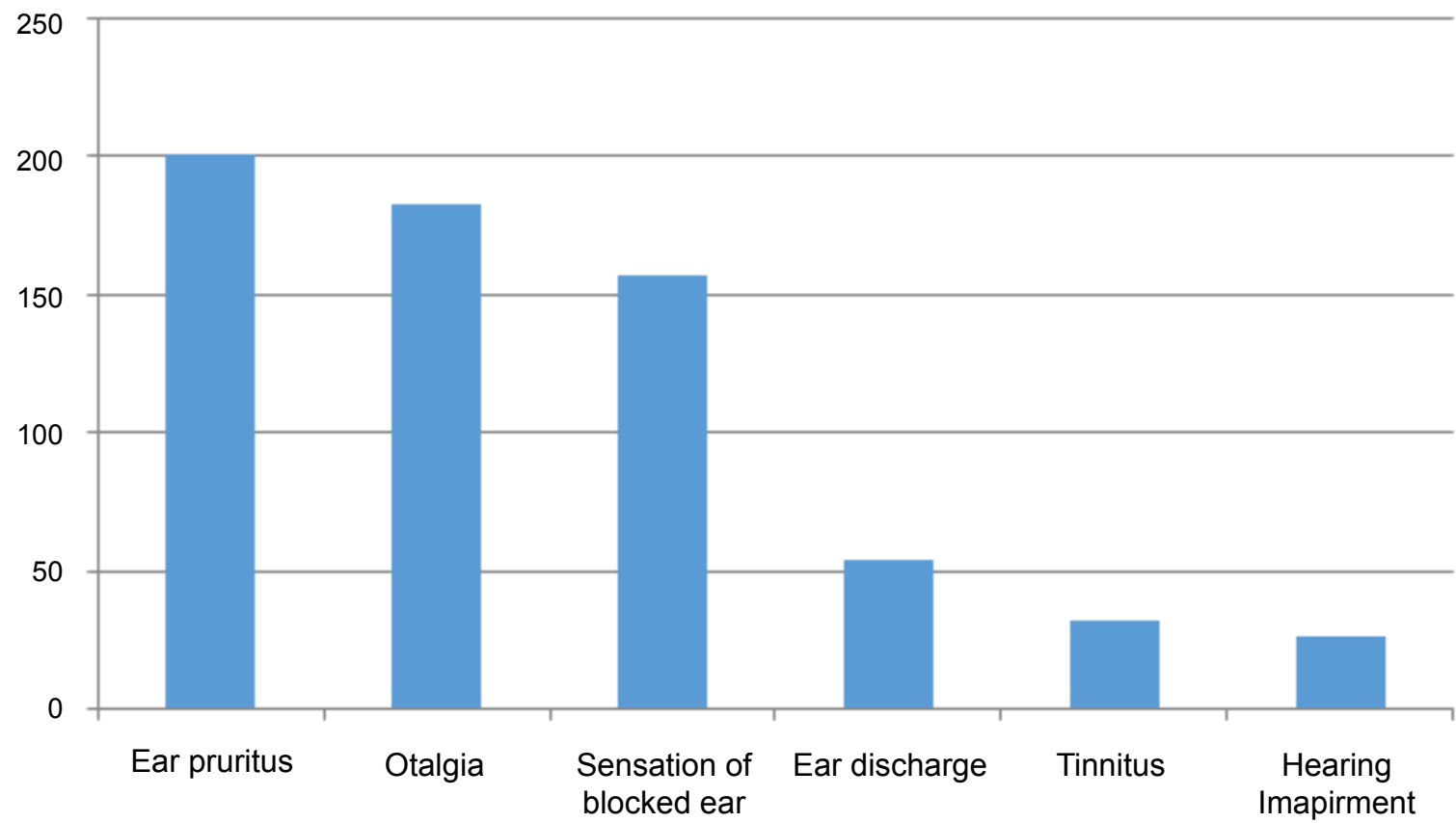

Figure 3: Clinical Presentations of the study population.

commonly associated risk factor was the use of antibiotic ear drops observed in $57 \%$ followed by the installation of mustard oil in $52 \%$ and swimming practiced by $19.6 \%$ of the total study population (Figure 2 ).

The most common presenting symptom in the study population was pruritus of the ear reported by $73 \%$ followed by otalgia in $66.5 \%$, sensation of the blocked ear in $57 \%$, discharge from the ear in $19.6 \%$, tinnitus in $11.6 \%$ and hearing impairment observed in $9.8 \%$ of the total study group (Figure 3 and Figure 4).

Fungi were observed to be the most common implicated pathogen in the majority of the cases of oto- mycosis. Fungi were isolated from 270 out of the total 275 samples obtained from clinically diagnosed cases of otomycosis leading to a fungal isolation rate of $98 \%$ in the total study group. The most commonly isolated fungi both from single and dual growth samples were Aspergillus seen in 250 out of 275 total samples making $91 \%$ of the total fungi isolated. The most common species of Aspergillus isolated was Aspergillus niger in 56\% of the total samples followed by Aspergillus fumigatus in $19.3 \%$ and Aspergillus flavus in $16 \%$. Other fungi isolated from the samples in order of the most common to least common were: Candida in $13.8 \%$, Mucor in $1.8 \%$, Penicillium in $1.1 \%$ and Trichophyton Mentagrophyte in 


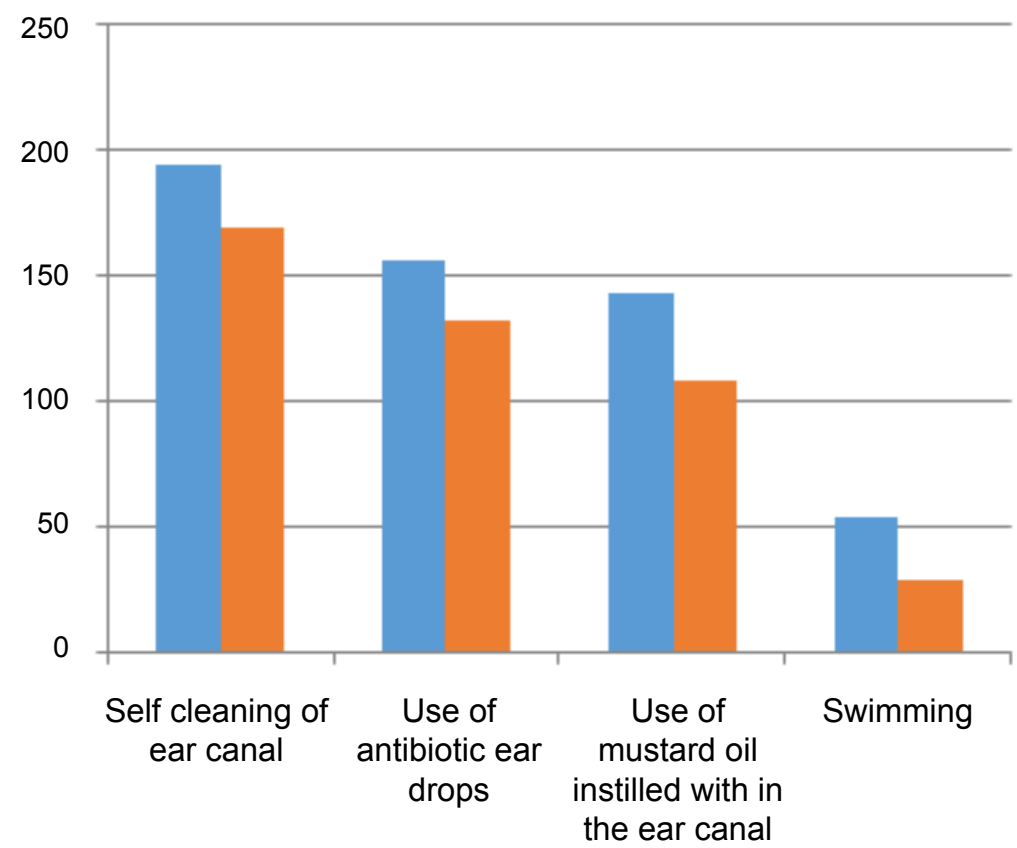

Risk Factors

Positive cases of otomycosis

Figure 4: Relation between risk factors and positive cases of otomycosis.

Table 2: Fungi and bacteria isolated from the study population.

\begin{tabular}{|c|c|c|c|c|}
\hline Fungi isolated from samples & \multicolumn{2}{|l|}{ Number of positive samples $(\mathrm{N}=\mathbf{2 7 5}$ ) } & \multicolumn{2}{|c|}{$\begin{array}{l}\text { Bacterial isolates found in association } \\
\text { with fungal isolates }\end{array}$} \\
\hline \multirow[t]{4}{*}{ Aspergillus Niger Complex } & \multirow{4}{*}{\multicolumn{2}{|c|}{137}} & Staphylococcus aureus & 30 \\
\hline & & & Pseudomonas aeruginosa & 14 \\
\hline & & & Klebsiella spp & 12 \\
\hline & & & Proteus spp & 5 \\
\hline \multirow[t]{2}{*}{ Aspergillus Fumigatus Complex } & \multirow{2}{*}{\multicolumn{2}{|c|}{42}} & Staphylococcus aureus & 22 \\
\hline & & & Pseudomonas aeruginosa & 11 \\
\hline \multirow[t]{2}{*}{ Aspergillus Flavus Complex } & \multirow{2}{*}{\multicolumn{2}{|c|}{36}} & Staphylococcus aureus & 18 \\
\hline & & & Pseudomonas aeruginosa & 8 \\
\hline \multirow[t]{2}{*}{ Candida Albicans } & \multirow{2}{*}{\multicolumn{2}{|c|}{11}} & Staphylococcus aureus & 6 \\
\hline & & & Klebsiella spp & 4 \\
\hline Candida Tropicalis & \multicolumn{2}{|l|}{8} & Klebsiella spp & 2 \\
\hline Candida Krusei & \multicolumn{2}{|l|}{7} & Klebsiella spp & 2 \\
\hline Mucor & \multicolumn{2}{|l|}{4} & Staphylococcus aureus & 2 \\
\hline Penicillum & \multicolumn{2}{|l|}{3} & & \\
\hline Trichophyton Mentagrophyte & \multicolumn{2}{|l|}{2} & & \\
\hline \multirow[t]{2}{*}{ Mixed fungal isolates } & $\begin{array}{l}\text { Mixed isolates containing two fungi } \\
\text { (Total Aspergillus spp }=32 \text { and } \\
\text { Candida spp }=11 \text { with Asperigillus } \\
\text { niger }=15, \text { Aspergillus fumigatus = } \\
10, \\
\text { Aspergillus flavus }=8 \text { ) }\end{array}$ & 18 & $\begin{array}{l}\text { Staphylococcus aureus } \\
\text { Pseudomonas aeruginosa } \\
\text { Klebsiella spp }\end{array}$ & $\begin{array}{l}12 \\
4 \\
2\end{array}$ \\
\hline & $\begin{array}{l}\text { Mixed isolates containing three } \\
\text { fungi (Total Aspergillus spp }=3 \text { with } \\
\text { Aspergillus niger }=2 \text {, Aspergillus } \\
\text { fumigatus }=1 \text { and Candida tropicalis } \\
=1 \text { and Mucor }=1\end{array}$ & 2 & Staphylococcus aureus & 1 \\
\hline \multicolumn{2}{|l|}{ Total number of fungal isolates } & 270 & Total number of bacterial isolates & 155 \\
\hline
\end{tabular}

$0.72 \%$ of the total 275 study group. Bacteria were isolated from 155 out of the total 275 samples (56.4\% of the total), where the most commonly isolated bacteria was Staphylococcus aureus seen in 58\% of the total 155 samples followed by Pseudomonas aeruginosa in $23.9 \%$ and Klebsiella spp. in $14.2 \%$ of the total bacterial sam- 
ples. Dual fungal growth was observed in $6.5 \%$ and triple fungal growth was seen in $0.72 \%$ of the total samples. In dual fungal growth most commonly co-isolated bacteria was Staphylococcus aureus seen in $66.7 \%$ of the 18 total samples while no bacteria were isolated in samples with triple fungal growth (Table 2 and Figure 5).

\section{Discussion}

Otomycosis is a highly prevalent fungal infection of the ear observed in many parts of the world [15]. Fungi are ubiquitous and are found around the globe, however, in regions such as Ekiti state a higher incidence of fungi can be expected due to environmental concerns such as ongoing construction and improper waste disposal that can not only provide habitat for fungi growth in the form of rotting plant and vegetable material but also means for fungi dispersal in the form of dust particles [16].

Our results revealed that the majority of the patients affected by otomycosis belonged to ages between 20 and 30 years amounting up to $38.2 \%$ of the total population followed by $28.4 \%$ lying in 10 to 20 years age group. This is consistent with the age groups defined by other studies such as in a study by R Mgbe, et al. in which out of the 338 patients diagnosed with otomycosis, $33.8 \%$ of the patient population lied in the $21-30$ years age group, making it the most prevalent age group of the study [17].

In our study population, $56 \%$ were males while $44 \%$ were females. The higher prevalence of otomycosis in males has been observed in various other studies such as by Kaur, et al. in the year 2000 [10] that reported 60\% of the population to be males, by Yehia, et al. in the year
1990 [18] who reported $52.5 \%$ to be males and by Ho, et al. in 2006 [6] and who found $56 \%$ of the study population to be males. However, this is in contrast to some other studies that revealed females to have higher cases of otomycosis such as that observed by Zaror, et al. who reported $65 \%$ of the study group to be females [5].

The majority of the patients reported unilateral ear involvement, as seen in 267 out of the total 275 patients with the right ear being involved in $82 \%$ of the cases. This is also similar to other studies that report a major chunk of unilateral ear involvement with the right side being more common possibly because it is on side of the dominant hand $[18,19]$. Nevertheless, bilateral involvement can be seen in immunodeficient cases such as in patients with AIDS and cancer [20]. We observed bilateral involvement in only 8 patients.

In our study, the three most common presenting symptoms were pruritus of the ear reported by $73 \%$, otalgia reported by $66.5 \%$ and, the sensation of blocked ear seen in $57 \%$. Other studies have also shown similar presenting symptoms with pruritus being the most common in a study by Aneja KR, et al. [19] and hearing loss being the most common symptom in $77.7 \%$ of the study population in an analysis by Khurshid Anwar, et al. [21].

All patients were questioned about possible risk factors that may predispose them to otomycosis. The most prevalent risk factor observed in the study population was self-cleaning of ears with objects such as q-tips, wooden sticks, metal pickers, hairpins and various other objects observed in $71 \%$ of the study population. Several studies have revealed an association between

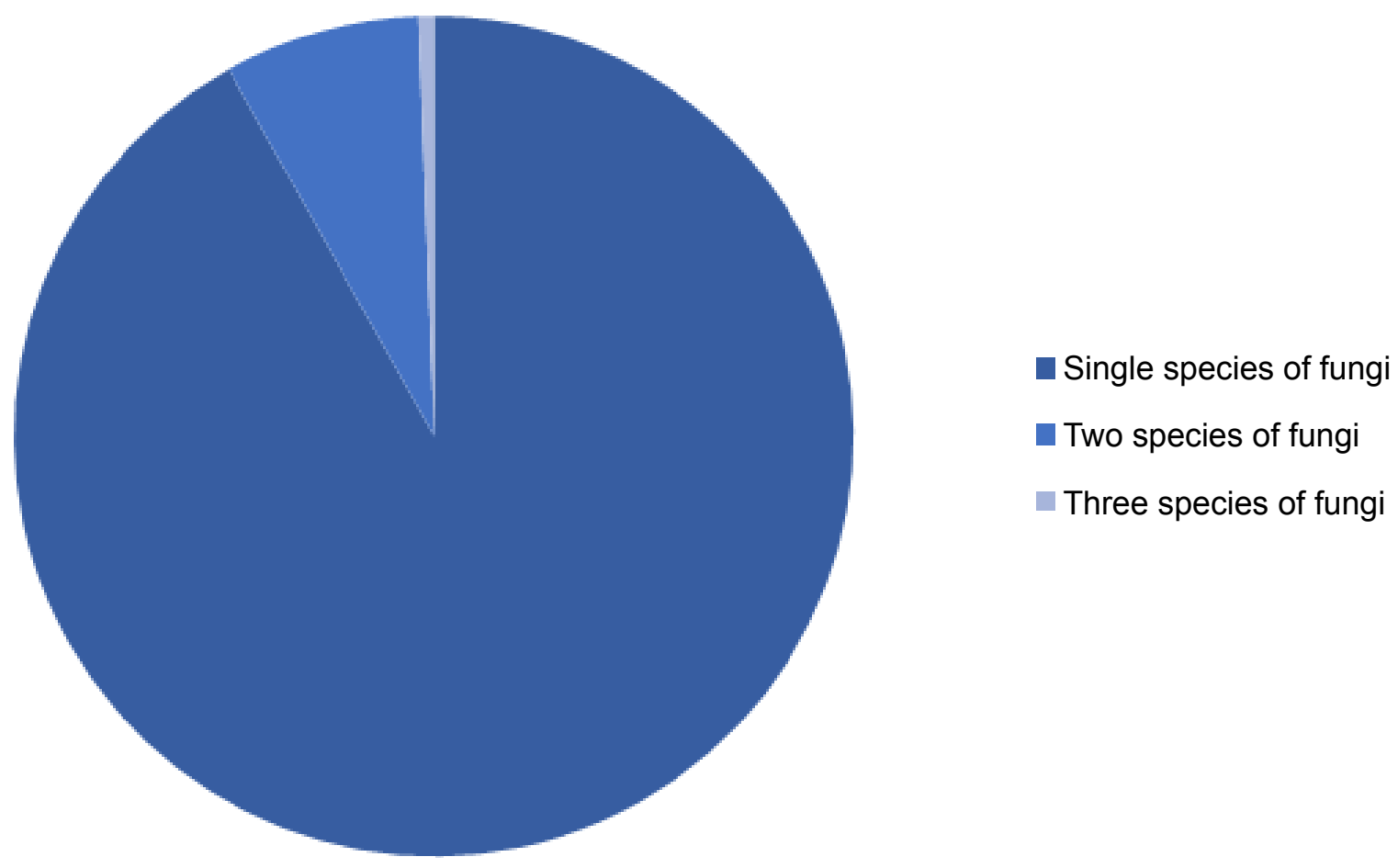

Figure 5: Fungal growth in samples. 
self-cleaning and ear infections such as in a study conducted by Priti Agarwal, et al. that reported self-cleaning to be the culprit in 224 out of the total 350 cases of otomycosis [22]. This can be explained by the removal of cerumen through the insertion of objects in the ear, which is a secretion of sebaceous and apocrine glands, and functions to protect the ear from invading organisms such as fungi and bacteria.

The second most commonly associated risk factor was the use of antibiotic ear drops observed in $57 \%$ followed by the instillation of mustard oil in $52 \%$ (Figure 2). Antibiotic ear drops can eliminate the natural flora of the ear and thus predispose the ear to local infections [23]. Mustard oil has also been reported as a common risk factor for ear infections as seen in a study by Pradhan, et al. that reported a significant association between otomycosis and mustard oil often used to relieve itching in the ear [4].

We also observed the practice of swimming in 54 of the total diagnosed cases of otomycosis. Swimming has been observed to be a predisposing factor for otomycosis as seen in a study conducted in Iran that reported a prevalence of swimming in otomycosis cases ranging from 5.7 to $81 \%$, with a mean value of $51.3 \%$, with higher prevalence recorded in the summer season [24].

Out of the total 275 samples, fungi were isolated in 270 samples, with a high isolation rate of $98 \%$. Aspergillus was observed in $91 \%$ of the total isolates. High fungal isolates have been reported by other studies such as $74.7 \%$ by Prasad SC, et al. and even $100 \%$ in a study by Gregson, et al. [15,25]. Aspergillus has also been reported as the most common constituent in other studies such as in a study conducted in Saudi Arabia that reported a frequency of Aspergillus isolation of 51.5\% [26] and $44.8 \%$ in a study conducted in Turkey [27]. The most common species of Aspergillus observed in our study is Aspergillus niger similar to other studies [15]. Some studies, however, reported other species of Aspergillus such as Fumigatus and Flavus to be the most commonly implicated fungi such as by Kaur R, et al. in 2000 [10]. In a few studies, candida was reported to be the most common organism isolated in cases of otomycosis. This can be seen in a study conducted in Ibadan where Candida was isolated in 15 out of the total 53 total isolates making $28.3 \%$ of the total [28].

Aspergillus is a ubiquitous mold identified as the causative organism in various infections and diseases. Aspergillus can be found in the form of minute conidia that can be easily propelled into the air with dust and other particles, making this its primary route of transmission [19]. In this study, identification and species division of Fungi and bacteria was done in accordance with the rules of the International Code of Botanical Nomenclature [29]. Identification of causative fungi in otomycosis is of prime significance so antifungal thera- py can be tailored to address the specific causative organism. Culture is ideal for isolating the organism but is often cumbersome and time-consuming. In this regard, several types of research have been conducted to use advanced morphology-based identification [30].

We observed bacterial growth in 155 out of a total of 275 samples. Mixed bacterial and fungal growths have been reported in several studies in as many as $6 \%$ of the total cases [31]. These infections can be the result of bacterial superinfection and present the additional trouble of recurrence and are difficult to cure. Recurrence can be attributed to the formation of biofilms [32] demonstrated by both Candida and Aspergillus spp. [33].

In our study, the most commonly isolated bacteria were identified as Staphylococcus aureus seen in 58\% of the total bacterial isolates followed by Pseudomonas aeruginosa in $23.9 \%$. This has been observed in other studies and the association between Aspergillus and Staphylococcus aureus has been attributed to the antibiotic activity of $A$. fumigatus against $S$. aureus [1]. In cases where mixed bacterial and fungal infections are reported, it becomes pertinent to prescribe both antifungal and antibiotic agents, further stressing the importance of knowing the causative organism of otomycosis, to narrow the instituted treatment.

\section{Conclusion}

From our study, we conclude that otomycosis is more prevalent between the ages of 20-30 years and in males. Fungi are the most commonly implicated organisms particularly Aspergillus and Candida and bacterial contamination should be expected in a portion of these cases especially by Staphylococcus aureus. More importantly, education about predisposing factors needs to be provided to stop the general population from using unhygienic objects for self-cleaning of ears, overuse of antibiotic ear drops and to curb the practice of instilling mustard oil into the ears. If proper attention is given to these risk factors, a significant drop in the incidence and prevalence of otomycosis can be expected.

\section{Acknowledgment}

We wish to thank all the Staffs of Federal Teaching Hospital Ido-Ekiti that contributed to the development of this research.

No conflict of interest among the authors or with anybody.

The manuscript is read and approved by the authors and it represents honest work.

\section{References}

1. Mugliston T, O'donoghue G (1985) Otomycosis--a continuing problem. J Laryngol Otol 99: 327-333.

2. Stern JC, Lucente FE (1988) Otomycosis. Ear Nose Throat J 67: 804-805 
3. da Silva Pontes ZB, Silva AD, de Oliveira Lima E, de Holanda Guerra M, Oliveira NM, et al. (2009) Otomycosis: A retrospective study. Braz J Otorhinolaryngol 75: 367-370.

4. Pradhan B, Tuladhar NR, Amatya RM (2003) Prevalence of otomycosis in outpatient department of otolaryngology in Tribhuvan University Teaching Hospital, Kathmandu, Nepal. Ann Otol Rhinol Laryngol 112: 384-387.

5. Zaror L, Fischman O, Suzuki FA, Felipe RG (1991) Otomycosis in Sao Paulo. Rev Inst Med Trop Sao Paulo 33: 169-173.

6. Ho T, Vrabec JT, Yoo D, Coker NJ (2006) Otomycosis: Clinical features and treatment implications. Otolaryngol Head Neck Surg 135: 787-791.

7. Paulose KO, Al Khalifa S, Shenoy P, Sharma RK (1989) Mycotic infection of the ear (otomycosis): A prospective study. J Laryngol Otol 103: 30-35.

8. Jackman A, Ward R, April M, Bent J (2005) Topical antibiotic induced otomycosis. Int J Pediatr Otorhinolaryngol 69: 857-860.

9. Sih T (2005) Otite externa. Passages de Paris 2: 166-171.

10. Kaur R, Mittal N, Kakkar M, Aggarwal AK, Mathur MD (2000) Otomycosis: A clinicomycologic study. Ear Nose Throat J 79: 606-609.

11. Jaiswal SK (1990) Fungal infection of ear and its sensitivity pattern. Indian Journal of Otolaryngology 42: 19-22.

12. Collee JG, Miles RS, Watt B (1996) Tests for identification of bacteria. Mackie and McCartney practical medical microbiology 14: 131-149.

13. Hurst WB (2001) Outcome of 22 cases of perforated tympanic membrane caused by otomycosis. J Laryngol Otol 115: $879-880$.

14. Chander J (2017) Textbook of medical mycology. JP Medical Ltd.

15. Prasad SC, Kotigadde S, Shekhar M, Thada ND, Prabhu P, et al. (2014) Primary otomycosis in the Indian subcontinent: Predisposing factors, microbiology, and classification. Int J Microbiol 2014: 636493.

16. Fasunla J, Ibekwe T, Onakoya P (2008) Otomycosis in western Nigeria. Mycoses 51: 67-70.

17. Mgbe R, Umana A, Adekanye A, Offiong M (2010) Otomycosis-A Management Challenge in Calabar, South-South Nigeria. The Internet Journal of Third World Medicine 9.

18. Yehia MM, Al-Habib HM, Shehab NM (1990) Otomycosis: A common problem in north Iraq. J Laryngol Otol 104: 387389.
19. Aneja KR, Sharma C, Joshi R (2010) Fungal infection of the ear: A common problem in the north eastern part of Haryana. Int J Pediatr Otorhinolaryngol 74: 604-607.

20. Viswanatha B, Sumatha D, Vijayashree MS (2012) Otomycosis in immunocompetent and immunocompromised patients: Comparative study and literature review. Ear Nose Throat J 91: 114-121.

21. Anwar K, Gohar MS (2014) Otomycosis; clinical features, predisposing factors and treatment implications. Pak J Med Sci 30: 564-567.

22. Agarwal P, Devi LS (2017) Otomycosis in a rural community attending a tertiary care hospital: Assessment of risk factors and identification of fungal and bacterial agents. $J$ Clin Diagn Res 11: 14-18.

23. Thrasher RD, Kingdom TT (2003) Fungal infections of the head and neck: An update. Otolaryngol Clin North Am 36: 577-594.

24. Gharaghani M, Seifi Z, Mahmoudabadi AZ (2015) Otomycosis in Iran: A review. Mycopathologia 179: 415-424.

25. Gregson AE, Touche CL (1961) Otomycosis: A neglected disease. J Laryngol Otol 75: 45-69.

26. Yassin A, Maher A, Moawad MK (1978) Otomycosis: A survey in the eastern province of Saudi Arabia. J Laryngol Otol 92: 869-876.

27. Ozcan KM, Ozcan M, Karaarslan A, Karaarslan F (2003) Otomycosis in Turkey: Predisposing factors, aetiology and therapy. J Laryngol Otol 117: 39-42.

28. Fayemiwo SA, Ogunleye VO, Adeosun AA, Bakare RA (2010) Prevalence of otomycosis in Ibadan: A review of laboratory reports. Afr J Med Med Sci 39: 219-222.

29. Gams W, Christensen M, Onions AH, Pitt JI, Samson RA (1985) Infrageneric taxa of Aspergillus. In: Samson RA, Pitt $\mathrm{JI}$, Advances in Penicillium and Aspergillus systematics. Plenum Press, New York, 55-62.

30. Balajee SA, Houbraken J, Verweij PE, Hong SB, Yaghuchi T, et al. (2007) Aspergillus species identification in the clinical setting Stud Mycol 59: 39-46.

31. Rama Rao SK, Manjaneyulu P (1979) Otomycosis. Indian J Otolaryngol 31: 65-68.

32. Philip A, Thomas R, Job A, Sundaresan VR, Anandan S, et al. (2013) Effectiveness of 7.5 percent povidone iodine in comparison to 1 percent clotrimazole with lignocaine in the treatment of otomycosis. ISRN Otolaryngol 2013: 239730.

33. Kakeya H, Imamura Y, Miyazaki T, Kohno S, Izumikawa $\mathrm{K}$, et al. (2011) Chronic fungal infection, up-to-date. Kansenshogaku Zasshi 85: 333-339. 